\title{
Correspondence
}

\section{Prophylactic epidural naloxone reduces the incidence and severity of neuraxial fentanyl-induced pruritus during labour analgesia in primiparous par- turients}

To the Editor:

Combined spinal epidural analgesia (CSEA) for labour pain is a widely accepted technique. However, pruritus is an undesirable complication when intrathecal or epidural narcotics are used in CSEA. Therefore, we tested the effect of a prophylactic epidural infusion of naloxone on fentanyl-induced pruritus, using a double-blinded randomized prospective design. The trial was approved by the Kitasato University Hospital's investigation committee on ethics and written informed consent was obtained from each patient.

Sixty-nine American Society of Anesthesiologists physical status I-II primiparous patients were enrolled. All patients received intrathecal analgesia with bupivacaine $2.5 \mathrm{mg}$, fentanyl $25 \mu \mathrm{g}$ and epinephrine $100 \mu \mathrm{g}$. Three minutes after the spinal injection, patients were randomized to one of two groups, and an epidural infusion was started at $10 \mathrm{~mL} \cdot \mathrm{hr}^{-1}$. Group NAL received $0.1 \%$ bupivacaine, $0.0002 \%$ fentanyl, $\mathrm{l}: 250,000$ epinephrine and $0.0004 \%$ naloxone epidurally. Group NS received the same solution without naloxone. The incidence and the maximum degree of pruritus, assessed by visual analogue itching score (VAIS), as well as the requirement for additional analgesics were noted.

There were no significant differences between the groups regarding maternal age, height, weight, the infant's gestational age, labour duration and mode of delivery. The total amount of additional bupivacaine in both groups was similar. However, the incidence of pruritus and VAIS in Group NAL was significantly less than in Group NS (Table).

We have demonstrated the effectiveness of epidural naloxone infusion on intrathecal and epidural fentanylinduced pruritus during labour analgesia. In contrast to the neurotoxicity of some opioids, ${ }^{1}$ naloxone may protect against opioid-induced neuronal damage. ${ }^{2}$ Basic experiments have shown that naloxone protects against inflammation-mediated degeneration or attenuates microglial activation. ${ }^{3}$ One could anticipate that nalox-
TABLE Maternal side effects and fetal adverse effects

\begin{tabular}{lll}
\hline & $\begin{array}{l}\text { Group NS, } \\
n=35\end{array}$ & $\begin{array}{l}\text { Group NAL, } \\
n=34\end{array}$ \\
\hline $\begin{array}{ll}\text { Motor block (number of patients) } \\
\text { 30 min after spinal analgesia }\end{array}$ & \\
Bromage 0 & $26(74 \%)$ & $24(71 \%)$ \\
Bromage 1-2 & $8(23 \%)$ & $10(29 \%)$ \\
$\quad$ Bromage 3 & $1(3 \%)$ & $0(0 \%)$ \\
Hypotension $(\%)$ & $4(11 \%)$ & $1(3 \%)$ \\
Nausea (\%) & $4(11 \%)$ & $3(9 \%)$ \\
Itching (\%) * & $25(71 \%)$ & $9(26 \%)$ \\
VAIS ** & $20(0,72)$ & $0(0,40)$ \\
Fetal deceleration $(n)$ & $11(31 \%)$ & $6(18 \%)$ \\
$\quad$ Severe variable deceleration $(n)$ & 9 & 6 \\
$\quad$ Prolonged deceleration $(n)$ & 3 & 3 \\
$\quad$ Late deceleration $(n)$ & 0 & 0 \\
\hline
\end{tabular}

Values except VAIS are number of patients (percentages, \%). Values for VAIS are median (range). VAIS = visual analogue itching score on a $100 \mathrm{~mm}$ scale. Severe variable and prolonged decelerations were observed in one and three patients in Groups NS and NAL respectively. * Significant difference $(P=0.0005)$ between the two groups. ** Significant difference $(P=0.0006)$ between the two groups.

one antagonizes the analgesic effect of opioids. However, a smaller dose of naloxone produced an analgesic effect in humans. ${ }^{4}$ Suggested explanations include: 1) the analgesia concentration response curve is far to the right of other opioid-related effects such as pruritus; 2) a small dose of naloxone may release endogenous endorphins at the postsynaptic receptor for analgesia or may act by inhibiting the presynaptic autoinhibition of enkephalins released by endogenous enkephalins itself. ${ }^{4}$ One may also be worried by the effects of naloxone on the fetus or neonate. One report showed that iv naloxone $40 \mu \mathrm{g}$ in neonates did not induce adverse effects. ${ }^{5}$ We observed no abnormal fetal heart rate pattern such as a loss of beat-to-beat variability in both groups. Therefore, the effects of naloxone on the fetus appear to be negligible.

Toshiyuki Okutomi MD

Miwako Saito MD

Junko Mochizuki MD

Kan Amano MD

Kanagawa, Japan 


\section{References}

I Kofke WA, Garman RH, Stiller RL, Rose ME, Garman $R$. Opioid neurotoxicity: fentanyl dose-response effects in rats. Anesth Analg 1996; 83: 1298-306.

2 Sinz EH, Kofke WA, Garman RH. Phenytoin, midazolam, and naloxone protect against fentanyl-induced brain damage in rats. Anesth Analg 2000; 91: 1443-9.

3 Lin $\Upsilon$, Qin L, Wilson BC, An L, Hong JS, Lin B. Inhibition by naloxone stereoisomers of beta-amyloid peptide (1-42)-induced superoxide production in microglia and degeneration of cortical and mesencephalic neurons. J Pharmacol Exp Ther 2002; 302: 1212-9.

4 Gan TJ, Ginsberg B, Glass PS, Fortney J, Jhaveri R, Perno R. Opioid-sparing effects of a low-dose infusion of naloxone in patient-administered morphine sulfate. Anesthesiology 1997; 87: 1075-81.

5 Wiener PC, Hogg MI, Rosen M. Effects of naloxone on pethidine-induced neonatal depression.

II-Intramuscular naloxone. Br Med J 1977; 2: 229-31.

\section{Spinal anesthesia for transurethral prostatectomy in a patient with multi- ple system atrophy}

To the Editor:

We would like to relate our opportunity to anesthetize a patient with Shy-Drager syndrome, a form of multiple system atrophy. A 76-yr-old man was scheduled for prostatectomy because of benign hypertrophy of the prostate. His neurodegenerative disease was diagnosed 14 years before when he presented with orthostatic hypotension, extrapyramidal rigidity, cerebellar ataxia, slight dysarthria, and sleep apnea. Fludrocortisone, theoepinephrine and the wearing of graded elastic stockings dramatically improved his hypotension. Blood pressure was $140 / 85 \mathrm{mmHg}$ and heart rate 72 beats. $\mathrm{min}^{-1}$. We offered him a regional rather than general anesthetic because of sleep apnea. He arrived unpremedicated in the operating room and became hypotensive while he was seated for spinal puncture. Spinal anesthesia was successfully achieved with $10 \mathrm{mg}$ of isobaric bupivacaine injected at the L2 to L3 level. Loss of sensation to pinprick reached $\mathrm{T} 2$ and paralysis of the lower limbs was complete. Anesthesia returned to the L2 level in $120 \mathrm{~min}$, and the total duration of the motor effects lasted three hours. The time course of hemodynamic variables is reported in the Figure. Hypertension was recorded throughout the surgical procedure that lasted two hours in the lithotomy posi-

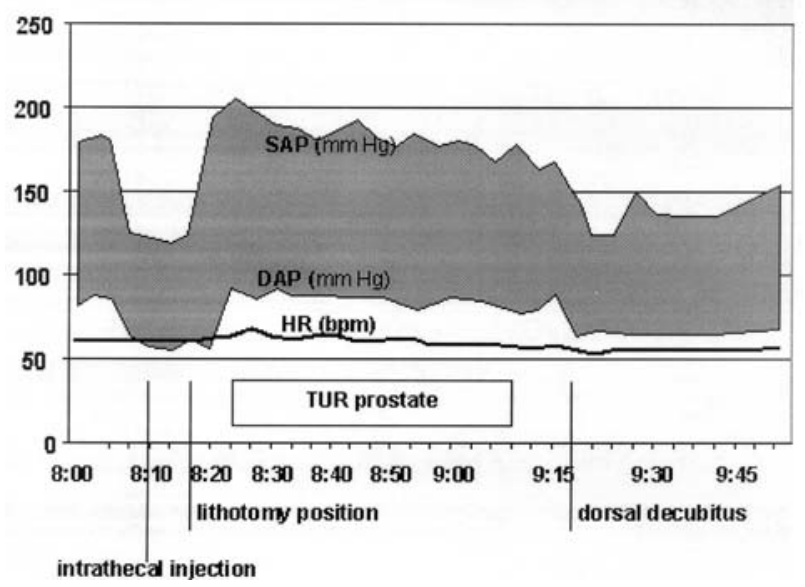

FIGURE Time-course of hemodynamic variables in a patient with multiple system atrophy receiving intrathecal bupivacaine $(10 \mathrm{mg})$ for transurethral resection of the prostate. $\mathrm{SAP}=$ systolic blood pressure, $\mathrm{DAP}=$ diastolic blood pressure and $\mathrm{HR}=$ heart rate.

tion. The blood pressure returned to preoperative values when the patient was placed supine after surgery. No vasopressors were required, and the patient was discharged home five days later.

Perioperative management of such patients remains critical. Disturbances in autonomic and central nervous systems result in defective baroreflex and vasoconstriction of both capacitance and resistance vessels, with an unpredictable response to vasoconstrictor agents in case of hypotension. Despite several cases of hypotension reported, general anesthesia is considered the gold standard in multiple system atrophy. When regional anesthesia was used, patients required high doses of local anesthetics and cephalad spread of anesthesia was almost restricted to the lumbar segments of the spinal cord. ${ }^{1-3}$ However, an interindividual variability in the dose-response to these agents or technical problems cannot be discarded in these reports. In the patient described, we used an anesthetic technique routinely performed in our institution. Short onset time and duration of anesthesia allowed surgery, and variations in blood pressure were hypotension during spinal puncture and hypertension throughout surgical procedure. In marked contrast to others, we found that the dose required to provide satisfactory anesthesia in this patient was not different than in healthy individuals. We conclude that spinal anesthesia can be suitable in such patients, that neuropathy is not worsened by anesthesia, and that a high level of sensory block does not induce hypotension. 